\title{
Chronic HCV Infection with Cryoglobulinemia, Associated with Cutaneous Subacute Lupus
}

\author{
Entela Shkodrani ${ }^{1 *}$, Silvan Frangaj ${ }^{2}$, Amarda Cenko $^{1}$ and Alert Xhaja ${ }^{1}$ \\ ${ }^{1}$ University Hospital Center "Mother Theresa", Tirana, Albania \\ ${ }^{2}$ Hospital of Shkodra, Albania
}

\begin{abstract}
Background: Chronic infection with Hepatitis C Virus (HCV): leading cause of chronic hepatitis, cirrhosis, liver cancer, primary indication for liver transplantation. HCV can give extrahepatic clinical manifestations and simulate autoimmune, rheumatologic and dermatologic diseases.

Case Report: Female patient, 34 y.o, lingering cutaneous eruption with itching and burning. Malar facial rash and papulo-squamous lesions on the back, chest and upper limbs. History of: Chronic HCV Infection, Mixed Cryoglobulinemia type II, Membranoproliferative Glomerulonephritis Convulsive episodes, Lyell Syndrome, Pulmonary Vessels Vasculitis , Recurrent Infections, Lupus-like Cutaneous Eruptions, Sjogren Sydrome-like clinical manifestations, Hemochroamtosis: Ferroportin Hzg. Mutation. Lab Results: Positive Antinuclear Antibodies, Extracted Nuclear Antigens, Cryoglobulins, Positive: Anti-HCV, Viral Load 4.800.000 copies/ml, Genotype 1b. Low C3 \& C4, Anemia, Leukopenia, Albuminuria \& Microhematuria, Hypoalbuminemia. Cutaneous Biopsy: Cutaneous Subacute Lupus.

Conclusion: Chronic HCV infection may be associated with clinical extrahepatic manifestations which can be interpreted incorrectly. This case reflects the ability of HCV to imitate a Cutaneous and Systemic Lupus Erythematosus that easily could have been wrongly diagnosed and mismanaged. Close cooperation between the relevant specialists in such case is essential. HCV infection can be added in the list of possible differential diagnoses of an Acute/Subacute Cutaneous Lupus Erythematous.
\end{abstract}

\section{Keywords: HCV, Acute/ Subacute Lupus, Cryoglobulinemia,}

\section{Introduction}

Chronic HCV infection affects 180 million people worldwide. It is one of the main causes of chronic hepatitis, cirrhosis, liver cancer and primary indication for liver transplantation. Moreover, it is able to induce extrahepatic clinical manifestations and simulate autoimmune, rheumatologic and dermatological diseases. Cutaneous Lupus appearing on the ground of chronic HCV infection constitutes an important clinical situation, as the signs and symptoms that may appear may wrongly point us to the diagnosis of Systemic Lupus and inappropriate management and therapy.

In the following, we are presenting the case of a chronic HCV patient, with concomitant Cryoglobulinemic vasculitis and renal impairment, hospitalized in the Dermatology Departament of University Hospital Centre "Mother Tereza" with Subacute Cutaneous Lupus.

\section{Case Report}

Female patient M.M, 34 y.o, presents in hospital with cutaneous eruption with symptoms of pruritus, burn and itching. During the general physical examination is noticed malar facial rash and papulo-squamous elements distributed mainly in the sunlight exposed areas of upper limbs, but also over the trunk and slightly on lower limbs. She refers a 5-months history, initially with the appearance of cutaneous facial elements. The disease was initially treated with topical cortisone, and the patient was advised to avoid sun exposure and to use photoprotection cream with SPF $50+$.

The patient presents a diverse and complex clinical history. In 2011 she was diagnosed with Membranoproliferative Glomerulonephritis, Type II Chronic Cryoglobulinemia and virus $\mathrm{C}$ chronic Hepatitis(HCV). After being diagnosed, she was treated with Rituximab and systemic corticotherapy for Membranoproliferative Glomerulonephritis associated with Mixed Cryoglobulinemia Type II and begins the therapy for HCV infection with peg-Interferon Alpha and Ribavirine.

The clinical history was accurately filed and complicated with: 1. Hypertensive episodes associated with a convulsive syncope for which was initiated the therapy with antihypertensive medication and Levetiracetam .2. Lyell Syndrome that was treated with bolus systemic corticotherapy and intravenous immunoglobulin, for which no possible cause was identified. 3. A pulmonary impairment with acute dyspnoea and pleural exudates, which was 
attributed to a vasculitis of pulmonary vessels, of which was set in resuscitation service .4. An urinary tract infection, purulent otitis media, esophageal candida and episodes of bacteremia as a result of immunosuppression by systemic corticosteroid therapy. 5. Suspected Hemocromatosis with high levels of ferritinemia and confirmed by positivity of a homozygous mutation in ferroportin.

In addition to the above mentioned complications, the patient was occasionally hospitalized for unclear dermatological manifestations (eruptions similar to Cutaneous Lupus ) and rheumatological manifestations (symptoms similar with Sjogren syndrome, positive Shirmer test, RF+, ANA+, Ro(SS-A)+ but no final conclusion has been given.

In our clinic the patient was diagnosed with Subacute Cutaneous Lupus with the suspition of LES. The systemic corticosteroid therapy (Prednisolone 50mg) and interdisciplinary consultations (Nephrologist, Rheumatologist, Neurologist and Gastro-hepatologist) were initiated together with relevant clinical and laboratory examinations.

The laboratory results are as follows:

1. ANA +++, ENA screen positive, Cryoglobuline,$+ \mathrm{C} 3$ and C4 decreased, Negative Anti-DsADN, Anticardiolipine negative, pANCA and cANCA negative.

2. anti-HCV positive, viral load 4.800 .000 copies / ml, HbsAg negative,
3. Leukopenia (3.300), Anemia (2.870.000, 8.3g / dl.), Ferritinemia and Sideremia at the lower limits of the range.

4. AST 59 and ALT 51.

5. Proteinuria with albuminuria (14 grams / 24 hours) and microhematuria, hypoproteinemia and hypoalbuminemia (4.4 and $2.3 \mathrm{~g} / \mathrm{dl}$ ) ( Uremia and creatinine were in normal range).

6. Cutaneous biopsy : Subacute Cutaneous Lupus

The systemic corticotherapy improved the dermatological manifestations. Consultation with the Neurologist suggested the gradual reduction of levetiracetam until complete discontinuation, as the clinical condition and history did not confirm the diagnose of epilepsy. Consultation with the Rheumatologist, despite the suggestive history, clinical and laboratory findings, excluded the presence of Systemic Lupus. The multidisciplinary consultation with the hepatologist and nephrologist decided that the patient should begin treatment for chronic HCV infection (genotype 1b) with the combination of Ledipasvir / Sofosvubir (Harvoni) after standard pegIFN and ribavirin treatment was already contraindicated as a result of previous failure and renal impairment. The patient left hospital with the diagnose of Subacute Cutaneous Lupus on the ground of HCV Chronic Infection to be followed by the hepatologist and nefrologist.
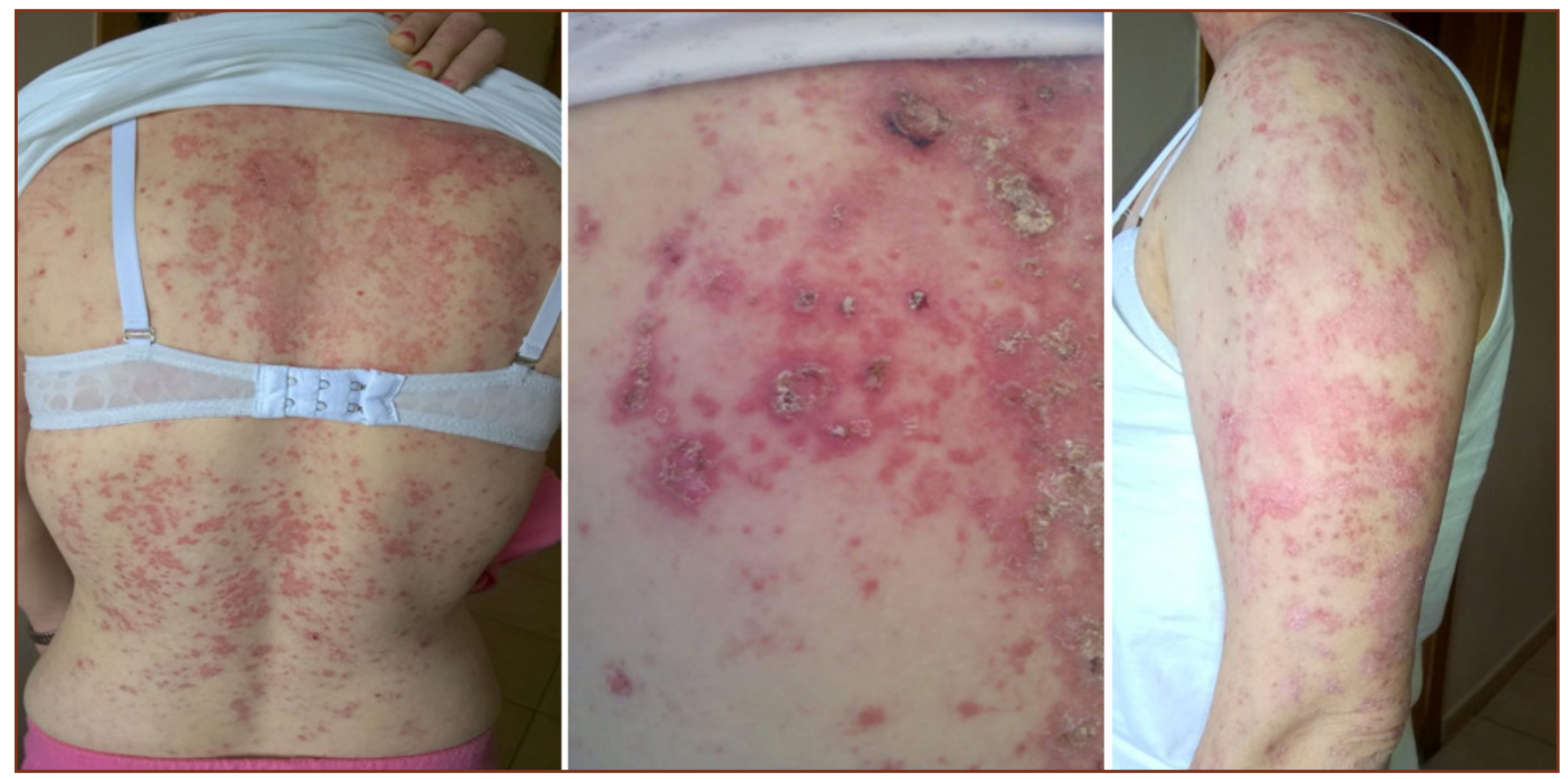

Fig. 1,2,3; Papulo-squamous elements distributed over the trunk and upper limbs. 


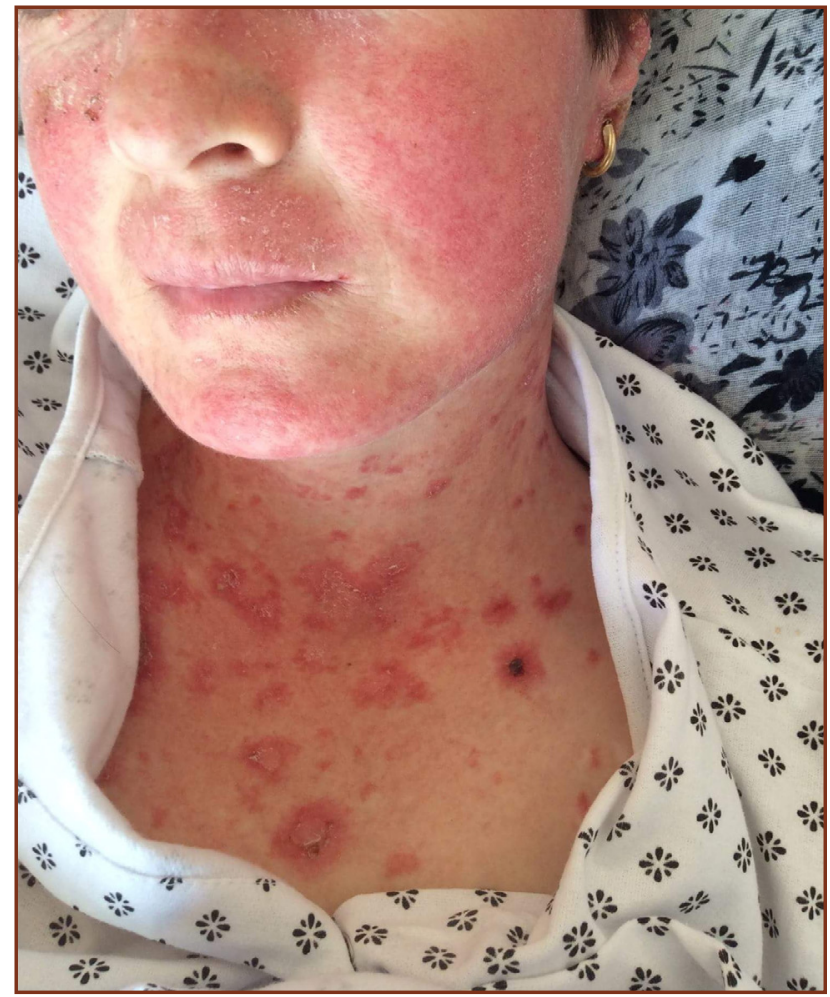

Fig. 4: Malar facial rash.

\section{Discussion}

$\mathrm{HCV}$ is a hepatotropic virus, the infection from which in most cases causes a chronic progressive fibrous hepatitis that slowly progresses to cirrhosis with hepatic insufficiency and significantly increases the risk of developing hepatocellular carcinoma. Before 1992 the predominant risk factors for being infected were the use of intravenous drugs, followed by blood transfusions, multiple sexual partners and medical manipulations. Besides liver disorders, chronic HCV infection is associated with extrahepatic manifestations such as rheumatic and autoimmune.

$\mathrm{HCV}$ has a persistent antigenic stimulation causing a clonal expansion of lymphocytes $\mathrm{B}$ which is associated with the production of autoantibodies (ANA, RF, Anticardiolipine, Cryoglobulin, Antithyroid Antibody, Anti-SMA, Anti-LKM ${ }^{(1)}$ which play a significant role in the pathogenesis of these clinical manifestations. It has now been established that chronic HCV infection is the main cause of Type II Mixed Cryoglobulinemia and can mimic, Sjogren Syndrome ${ }^{(2)}$ Rheumatoid Arthritis ${ }^{(3,4) \text {, }}$ Antiphospholipid Syndrome and Nodose Panarteritis ${ }^{(5)}$ and Systemic Erythematous Lupus ${ }^{(6)(7)}$. The prevalence of HCV presence in patients with Systemic Erythematous Lupus is higher than that in healthy population, therefore it has been suggested that during the diagnostic procedures for Systemic Lupus, should be performed the HCV tests, especially when the clinical background is suspicious, faint or not characteristic.

In addition, chronic $\mathrm{HCV}$ infection may be accompanied by special dermatological diseases that may be directly related to HCV's interactions to the body or come as a side effect of IFN therapy. Nowdays the conjuction between HCV and Lichen Planus, Porphyria Cutanea Tarda, Prurigos as well as Cutaneous Vasculitis and Chronic leg ulcers, related to Mellitus Diabetes type II is quite clear. IFN treatment may aggravate Psoriasis and Sarcoidosis as well as inducing total Alopecia.

Our patient was diagnosed with Subacute Cutaneous Lupus and based on the clinical and anamnestic background was also suspected of Systemic Erythematous Lupus. The diagnosis of Systemic Erythematous Lupus is based on eleven(11) criteria set by the American College of Rheumatology in 1982 and updated in 1997. The acquirement of at least 4 of them, serially or simultaneously,during any interval of observation confirms the disease. Our patient fulfilled three of these criteria (cutaneous manifestations, ANA +, proteinuria $>0.5 \mathrm{~g} / 24 \mathrm{~h}$ with hematuria), which in the first sight directed us toward a Systemic Erythematous Lupus. Discovering more in the patient history and the help of interdisciplinary consultations we concluded that an ensemble of these clinical manifestations actually results in chronic $\mathrm{HCV}$ infection. The appropriate treatment for such a case are the antiviral therapies agains $\mathrm{HCV}$, which address the key etiologic factor.

\section{Conclusion}

Chronic HCV infection may be associated by clinical extrahepatic manifestations that can be interpreted incorrectly. This case reflects the ability of HCV to imitate a cutaneous and systemic erythematous lupus syndrome that could easily be diagnosed incorrectly and mismanaged. Close cooperation between the relevant specialists in such case is essential. HCV infection should be added to the list of possible differential diagnoses of a Acute / Subacute Cutaneous Lupus that is associated with impairement of the internal organs, especially in cases that suggest epidemiological datas.

\section{Reference}

1. McMurray RW, Elbourne K. Hepatitis C virus infection and autoimmunity. Semin Arthritis Rheum 1997;26:689-701.

2. Garci'a-Carrasco M, Ramos-Casals M, Cervera R, Font J, Vidal J, Mun oz FJ, et al. Hepatitis C virus infection in "primary" Sjo"gren's syndrome: prevalence and clinical significance in a series of 90 patients. Ann Rheum Dis 1997;56:173-5. 
3. Rivera J, Garci'a-Monforte A. Hepatitis C virus infection presenting as rheumatoid arthritis. Why not? J Rheumatol 1999;26: 2062-3.

4. Espinosa-Morales R, Escalante A. Diagnostic confusion caused by hepatitis $\mathrm{C}$ : hemochromatosis presenting as rheumatoid arthritis. J Rheumatol 1998;25:2459-63.

5. Soufir N, Descamps V, Crickx B, Thibault V, Cosnes A, Becherel PA, et al. Hepatitis $\mathrm{C}$ virus infection in cutaneous polyarteritis nodosa: a retrospective study of 16 cases. Arch Dermatol 1999; 135:1001-2.

6. Ramos-Casals, M., Font, J., Garcia-Carrasco, M., Cervera, R., Jimenez, S., Trejo, O., \& De La Red, G. (2000). Hepatitis $\mathrm{C}$ virus infection mimicking systemic lupus erythematosus. Arthritis Rheum, 43(12), 2801-6.

7. Cohen P. Extrahepatic manifestations of hepatitis $\mathrm{C}$ virus. Presse Med 2000;29:209-14.

*Corresponding author:

Entela Shkodrani, Clinic of Dermatology, University Hospital Center "Mother Theresa" Tirana, Albania

Phone: 0355692081029

Email: entela.shkodrani@gmail.com

Financial or other Competing Interests: None. 